\title{
Right Upper-Quadrant Pain in a Patient with Drug Abuse, Secondary Syphilis and Occult Hepatitis B Virus
}

\author{
Cory M. Fielding ${ }^{a}$ Paul Angulo a, b \\ ${ }^{a}$ Department of Internal Medicine and ${ }^{b}$ Division of Digestive Diseases and Nutrition, University of Kentucky, \\ Lexington, Ky., USA
}

\section{Key Words}

Abdominal pain · Drug-induced hepatitis · Syphilis

\begin{abstract}
Objective: To describe the etiology of hepatitis and identify occult hepatitis B virus (HBV) infection. Clinical Presentation and Intervention: A 40-year-old man presented with severe abdominal pain and jaundice, a history of acute HBV infection that had cleared as well as the use of acetaminophen, methamphetamine, buprenorphine and marijuana. He admitted to having had unprotected sex with multiple partners of both genders. A thorough skin examination revealed papulosquamous lesions on his penis, scrotum, upper and lower extremities and feet. Transaminases and bilirubin were elevated. His rapid plasma reagin was reactive, and hepatitis serologies showed occult HBV. Liver biopsy showed severe hepatitis, but the stains for hepatitis B surface antigen and hepatitis $B$ core antigen were negative. The pathological findings were highly indicative of drug-induced hepatitis without evidence of chronic hepatitis, reactivation of HBV or syphilitic hepatitis. With supportive management and abstinence from drugs, his condition improved. Conclusion: This case describes a patient with multiple potential causes for
\end{abstract}

hepatitis and highlights the importance of obtaining a detailed social history. Further, one should consider the presence of occult HBV and recognize the serologic pattern.

(c) 2014 S. Karger AG, Basel

\section{Introduction}

Acute hepatitis has many independent and concomitant causes. When evaluating a patient with acute hepatitis, a clinician must investigate the most likely etiologies thoroughly. This case report chronicled the procedures that were followed to diagnose the causes of acute hepatitis in a patient with multiple potential causes.

\section{Case Report}

A 40-year-old Caucasian man presented to the Emergency Department in December 2012 with right upper-quadrant pain of 3 weeks' duration. His wife, who was present, had also noticed a yellowing of his sclera and skin over this period. The pain had started as an ache in his right side and had gradually increased in intensity to 8 on a severity scale of 0 to 10 . No exacerbating or alleviating factors were identified. He reported a history of buprenorphine/

\begin{tabular}{ll}
\hline KARGER & $\begin{array}{l}\text { Co 2014 S. Karger AG, Basel } \\
\text { 1011-7571/14/0235-0471 } \$ 39.50 / 0 \quad \text { Karger }\end{array}$ \\
$\begin{array}{l}\text { E-Mail karger@karger.com } \\
\text { www.karger.com/mpp }\end{array}$ & $\begin{array}{l}\text { This is an Open Access article licensed under the terms of the } \\
\text { Creative Commons Attribution-NonCommercial 3.0 Un- } \\
\text { ported license (CC BY-NC) (www.karger.com/OA-license), } \\
\text { applicable to the online version of the article only. Distribu- } \\
\text { tion permitted for non-commercial purposes only. }\end{array}$
\end{tabular}

Cory M. Fielding, MD

304B Charles T. Wethington Bldg.

900 South Limestone

Lexington, KY 40536 (USA)

E-Mail cory.fielding@uky.edu 


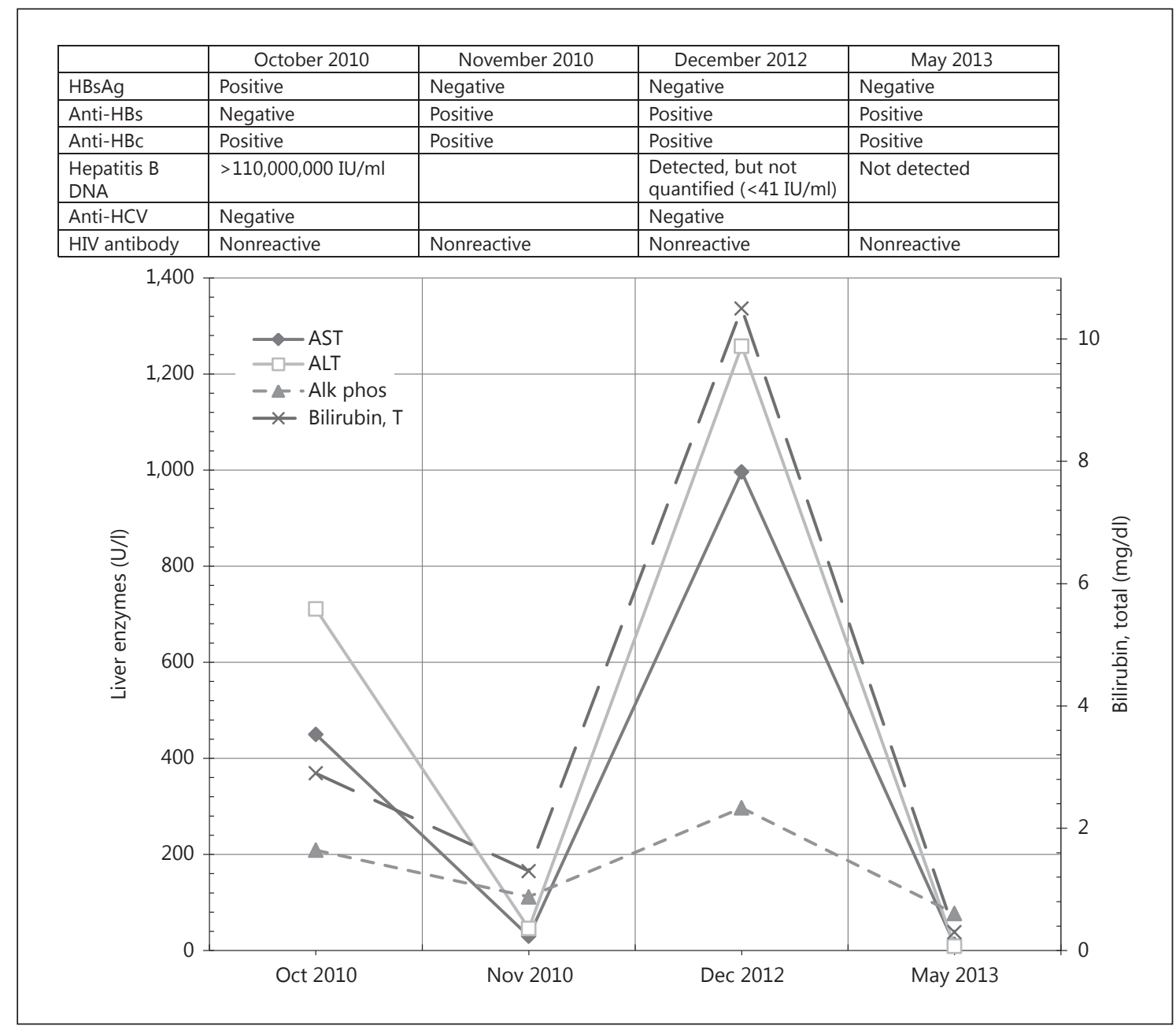

Fig. 1. Laboratory findings. Alk phos = Alkaline phosphatase; ALT = alanine aminotransferase; Anti-HBc = hepatitis B core antibody; Anti-HBs = hepatitis B surface antibody; Anti-HCV = hepatitis C antibody; AST = aspartate aminotransferase; HBsAg = hepatitis B surface antigen.

naloxone use years ago, but initially denied recent use. He complained of fatigue and malaise but denied experiencing nausea, vomiting, diarrhea, fever, chills or weight loss. He had not had contact with sick people or travelled recently. Tobacco, alcohol and intravenous drug use were initially denied. He claimed to be monogamous with his wife. He had worked as a welder for 15 years and had noticed some 'burns' on his upper extremities over the past few weeks and claimed he did not wear the proper protective clothing while welding.

The patient's medical history was significant for an acute hepatitis B virus (HBV) infection 2 years previously. He claimed to have acquired this from a tattoo while in prison. His transaminases had normalized a month later, with the follow-up hepatic panel showing clearance of the acute infection and he had been asymptomatic. He was negative for hepatitis $C$ virus in 2010 and 2012, for the period tested. He did not keep his follow-up clinic appointments after his clinical improvement from the acute HBV.
The physical examination revealed a thin, disheveled man in no apparent distress. He was afebrile with normal vital signs. His skin was jaundiced with numerous nontender papulosquamous lesions with superficial scales of $0.5-1 \mathrm{~cm}$ in diameter on his upper extremities (including the palms of his hands) in various states of healing. He had scleral icterus, poor dentition and a nontender ulcerated lesion of $7 \mathrm{~mm}$ in diameter on his soft palate. The abdominal examination revealed a tender, enlarged liver palpable to $6 \mathrm{~cm}$ below the right costal margin. A genital examination was refused by the patient. He was alert and oriented with no neurologic deficits or evidence of encephalopathy. Figure 1 lists the pertinent laboratory investigations conducted. An abdominal ultrasound showed a 'starry sky' appearance of the liver with intra- and extrahepatic biliary ductal dilatation without obstructing stones or mass.

Given the unusual skin and oral findings upon examination, additional history was taken from the patient when his family was not present. Although he had initially denied drug use, he eventu- 
Fig. 2. a Trichrome-stained low-power view of periportal fibrosis with inflammation. $\mathbf{b}$ High-power view of severe hepatitis with mixed inflammatory infiltrate of mostly lymphocytes and neutrophils, but eosinophils and plasma cells were also identified.
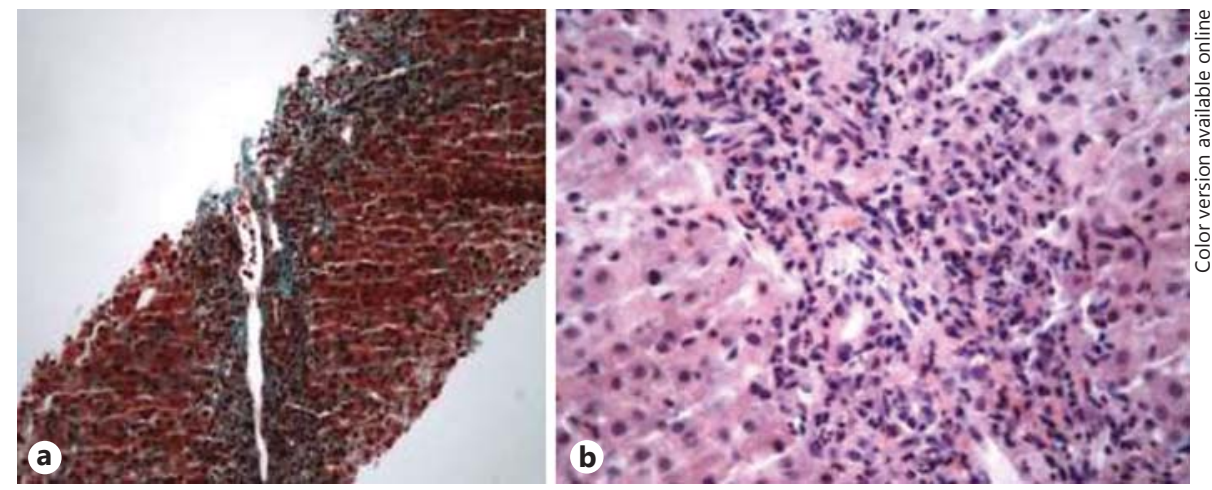

ally admitted to using methamphetamine, oxycodone/acetaminophen, buprenorphine and marijuana regularly and concomitantly over the past year. He reported a 20-year history of intravenous drug use that had ended 3 years previously. He admitted to having unprotected sex with multiple partners of both genders. He had noticed an enlarged inguinal lymph node (approximately $3 \mathrm{~cm}$ in diameter) a month before presentation that had resolved without ulceration. He then allowed for a more thorough skin examination, and we discovered similar papulosquamous lesions on his penis, scrotum, lower extremities and feet. Considering the new insight into his social history, more laboratory tests were collected. The patient's rapid plasma reagin was reactive with a titer of 1:512. Treponema pallidum particle agglutination was reactive as well. His wife was informed about this result, and she tested negative for syphilis. Acute HIV infection was suspected, but HIV antibody and quantitative viral load were not detected. Cytomegalovirus IgM and IgG antibodies were positive. The autoimmune hepatitis panel was negative and so were antibodies to hepatitis A, C, D and E. The differential diagnosis for his acute hepatitis included reactivation of HBV, syphilitic hepatitis, Cytomegalovirus hepatitis and drug-induced hepatitis.

The hepatitis B panel was not indicative of acute infection since the hepatitis B surface antigen was negative. A percutaneous liver biopsy was obtained (fig. 2). Severe Kupffer cell hypertrophy, marked ballooning degeneration of hepatocytes and centrilobular necrosis were present. Immunohistochemical stains for hepatitis B surface antigen and hepatitis B core antigen were negative. No spirochetes or granulomatous changes were seen. These pathologic findings were highly indicative of drug-induced hepatitis without evidence of chronic hepatitis, reactivation of HBV or syphilitic hepatitis.

The patient was given two doses of intramuscular penicillin $G$ benzathine of 2.4 million units and counseled to refrain from drug use and unprotected sexual contact. When his transaminases began trending down and his pain resolved, he was discharged home. He presented to the Infectious Disease Clinic for a 6-month follow up in May 2013 with a 64-fold decline in rapid plasma reagin titer to $1: 8$. HBV DNA was not detected, and his serologies indicated immunity. A urine drug screen showed no illicit drug use, and he claimed to have abstained from sexual contact for the past 6 months. A final diagnosis of drug-induced acute hepatitis from methamphetamine, oxycodone/acetaminophen and buprenorphine in the setting of secondary syphilis and an occult HBV infection was made.

Right Upper-Quadrant Pain with Drug Abuse, Syphilis and Occult HBV

\section{Discussion}

This interesting case chronicled the diagnostic process for a patient with a number of potential causes of acute hepatitis, demonstrating the importance of avoiding premature closure, which is the most common cognitive error when establishing a diagnosis [1]. In immunocompetent adults with acute $\mathrm{HBV}$ infection, 95\% spontaneously develop seroclearance of HBsAg, which indicates resolution of acute infection. Despite this clearance, small amounts of viral DNA may still be detected in the serum by PCR, demonstrating an inactive residual phase of infection [2]. Quantitative detection of HBV DNA is made typically by real-time PCR amplification and the detection of its genomic DNA being compared to a quantitative standard. The quantitative range for detection of this assay at our reference laboratory is $20-170,000,000 \mathrm{IU} /$ $\mathrm{ml}$. Given the multiple symptoms and medical history of our patient, our initial leading diagnosis was a reactivation of HBV. However, the serologies did not support an active HBV infection and the patient's social history and physical exam suggested additional etiologies. His hepatitis panel was consistent with an occult HBV infection since his hepatitis B surface antigen was negative. HBV DNA was detectable but too low for quantification by PCR, and hepatitis B core antibody and hepatitis B surface antibody were positive $[2,3]$. However, occult HBV is usually asymptomatic and is only detected when testing for other reasons such as sexually transmitted infections, immunosuppression or when considering therapy for hepatitis $C$ virus [4]. Areas for further research include identifying the prevalence and infectivity of occult HBV and the role it plays in fibrosis formation.

Secondary syphilis is a rare cause of hepatitis, usually with a mild clinical presentation. The typical findings are mildly elevated transaminases, normal or mildly elevated 
bilirubin and a disproportionately high serum alkaline phosphatase level [5]. Our patient's acute illness was not consistent with these levels, and his liver biopsy did not reveal spirochetes or granulomatous changes. However, in a large number of cases, there is no visualization of spirochetes or noncaseating granulomas on biopsy [6].

Drug-induced hepatic toxicity is the most common cause for withdrawing a drug from the market [7]. Our patient showed evidence of two active mechanisms of hepatic injury: direct hepatocellular toxicity and cholestasis. He admitted to taking acetaminophen, methamphetamine and buprenorphine, all of which are known to deplete glutathione levels, leaving hepatocytes susceptible to oxidative damage which prevents mitochondria from synthesizing adenosine triphosphate, resulting in necrosis $[8,9]$. However, hepatic damage by amphetamines is most commonly seen with methylenedioxymethamphetamine (Ecstasy). Because of the unregulated manufacturing of illicit substances, such as methamphetamine, a patient may also be at risk of ingesting hepatotoxic contaminants [10]. Withdrawal of the offending agent(s) and supportive care is the treatment of choice for drug-in- duced hepatitis. We would like to note that a major limitation of this report was the sporadic follow-up appointments.

\section{Conclusion}

This case highlighted the diagnostic process for a patient presenting with multiple potential causes for abdominal pain as well as the importance of taking a detailed social history. Further, one should consider the presence of occult HBV and recognize the serologic pattern. Our patient's presentation of secondary syphilis was compelling, but it was the pathology and laboratory results that assisted us in making the diagnosis of drug-induced hepatitis being the most likely cause of his symptoms.

\section{Disclosure Statement}

The authors declare they have not received any financial support with respect to the authorship and publication of this article and have no potential conflicts of interest.

\section{References}

1 Graber ML, Franklin N, Gordon R: Diagnostic error in internal medicine. Arch Intern Med 2005;165:1493-1499.

2 Said ZN: An overview of occult hepatitis B virus infection. World J Gastroenterol 2011;17: 1927-1938.

3 Kaminski G, Alnaqdy A, Al-Belushi I, et al: Evidence of occult hepatitis B virus infection among Omani blood donors: a preliminary study. Med Princ Pract 2006;15:368-372.

4 Schmeltzer P, Sherman KE: Occult hepatitis B: clinical implications and treatment decisions. Dig Dis Sci 2010;55:3328-3335.
-5 Lin H, Russo P, Rook M: Secondary syphilitic hepatitis in a 17-year-old girl. J Pediatr Gastroenterol Nutr 2012;55:e134-135.

6 6 Young MF, Sanowski RA, Manne RA: Syphilitic hepatitis. J Clin Gastroenterol 1992;15: 174-176.

7 Lee WM: Drug-induced hepatotoxicity. N Engl J Med 2003;349:474-485.
8 Hinson JA, Roberts DW, James LP: Mechanisms of acetaminophen-induced liver necrosis. Handb Exp Pharmacol 2010;196:369405

9 Berson A, Fau D, Fornacciari R, et al: Mechanisms for experimental buprenorphine hepatotoxicity: major role of mitochondrial dysfunction versus metabolic activation. J Hepatol 2001;34:261-269.

10 Jones AL, Simpson KJ: Mechanisms and management of hepatotoxicity in ecstasy (MDMA) and amphetamine intoxications. Aliment Pharmacol Ther 1999;13:129-133. 